Acta Technologica Agriculturae 3

Nitra, Slovaca Universitas Agriculturae Nitriae, 2017, pp. 78-84

\title{
APPROXIMATIONS TO THE NON-ISOTHERMAL DISTRIBUTED ACTIVATION ENERGY MODEL FOR BIOMASS PYROLYSIS USING THE RAYLEIGH DISTRIBUTION
}

\author{
Alok DHAUNDIYAL*1 ${ }^{*}$ Suraj B. SINGH² \\ ${ }^{1}$ Szent István University, Gödöllő, Hungary \\ ${ }^{2}$ Govind Ballabh Pant University of Agriculture and Technology, Pantnagar, U. K, India
}

\begin{abstract}
This paper deals with the influence of some parameters relevant to biomass pyrolysis on the numerical solutions of the nonisothermal $n^{\text {th }}$ order distributed activation energy model using the Rayleigh distribution. Investigated parameters are the integral upper limit, the frequency factor, the heating rate, the reaction order and the scale parameters of the Rayleigh distribution. The influence of these parameters has been considered for the determination of the kinetic parameters of the non-isothermal $n^{\text {th }}$ order Rayleigh distribution from the experimentally derived thermoanalytical data of biomass pyrolysis.
\end{abstract}

Keywords: biomass pyrolysis; distributed activation energy model; non-isothermal kinetics; Rayleigh distribution; asymptotic expansion

The Distributed Activation Energy Model (DAEM) or Multiple Reaction Model (MRM) applies either to the total amount of volatiles released or to the amount of an individual volatile constituent like carbon monoxide or tar (Howard, 1981). It is also called the Distributed Rate Model, and uses Vand's treatment of independent parallel processes (Vand, 1943) in modelling the resistance of metallic films. The detailed study includes the total amount of volatiles released during the pyrolysis process (Howard, 1981; Donskoi and McElwain, 1999). Originally, the coal devolatilization, however, was considered first to develop the Distributed Activation Energy Model (Pitt, 1962), yet the DAEM also applies to the pyrolysis of other materials, including biomass, residual oils, resin char (Teng and Hsieh, 1999), and kerogen (Lakshmanan and White, 1994). Calculations of solutions to this model involve evaluations of double integrals, which vary rapidly and hence create significant numerical difficulties. In order to tackle the integral complication, asymptotic expansion has been adopted for the accurate approximation to our problem. The solution for the DAEM model is given by Eq. (1):

$$
v=\left\{\begin{array}{c}
\int_{0}^{\infty} \exp \left(-k_{0} \int_{0}^{t} e^{-\frac{E}{R T}} d t\right) \frac{E}{\beta^{2}} \exp \left(\frac{-E^{2}}{2 \beta^{2}}\right) d E, \\
\text { for the first order reaction } \\
\int_{0}^{\infty}\left[1-(1-n) k_{0} \int_{0}^{t} e^{-\frac{E}{R T}} d t\right]^{\frac{1}{(1-n)}} \frac{E}{\beta^{2}} \exp \left(\frac{-E^{2}}{2 \beta^{2}}\right) d E \\
\text { for the } n^{\text {th }} \text { order reaction }
\end{array}\right.
$$

where:

$T(t) \quad$ - the time varying absolute temperature of the biomass

$R$

- the universal gas constant
$E \quad$ - the activation energy

$k_{0} \quad$ - the pre-exponential or frequency factor

$\beta(\beta>0)$ - the scale parameter of initial distribution function respectively

The aim of this paper is to use asymptotic methods to make accurate approximations to the integrals and thereby evaluate the influence of relevant parameters on the biomass pyrolysis.

\section{Material and methods}

\section{Asymptotic expansion}

The relationship between the DAEM and the single first order reaction model have been explored by Niksa and Lau (1993), which is based on the approach of holding the activation energy fixed, and defining an effective or nominal rate constant $\langle k\rangle$ (Niksa and Lau, 1993). They also gave an analytical approximations to the DAEM for the exponentially and the linearly varying temperature profiles. The basis of approach lies on exploiting the rapid changes occurring in the double exponential term in Eq. (1). The procedure adopted by Niksa and Lau (1993) provides accurate approximations to the DAEM for all the relevant parameters for the kinetic mechanism of biomass pyrolysis. The modification done by them is simply refinement of the concept applied by Suuberg (1983), who used unit step function approximation to Double exponential term (DExp) (Howard, 1981; Vand, 1943; Pitt, 1962). In the subsequent sections, a more accurate approximation to DExp has been developed, which is implemented in the two different types of distribution cases (Narrow and Wide distribution). Engineering, Gödöllő, Hungary, e-mail: alok.dext@hotmail.com; Suraj B. Singh, Govind Ballabh Pant University of Agriculture and Technology, Department of Mathematics, Statistics \& Computer Science, Pantnagar, U. K, India, e-mail: drsurajbsingh@yahoo.com 


\section{Simplification of Double exponential term (DExp)}

The integrand in Eq. (1) contains two terms. The first term (DExp) depends on time through the temperature history experienced by the sample. The second part is independent of time and depends on the distribution of volatiles in the sample.

Approximation to the double exponential is represented as:

$$
D \operatorname{Exp}=\exp \left(-k_{0} \int_{0}^{t} e^{-\frac{E}{R T}} d t\right)
$$

In order to motivate the stepwise simplification of this integrand, some typical values of the parameters and functions are considered. The frequency factor or preexponential term is mainly in the range of $k_{0} \sim 10^{10}-10^{13} s^{-1}$, whereas the activation energies of interest are in the range from $100 \mathrm{~kJ} \cdot \mathrm{mol}^{-1}$ to $300 \mathrm{~kJ} \cdot \mathrm{mol}^{-1}$. The typical values of temperatures depend on the particular experiments, but $1000 \mathrm{~K}$ to $2000 \mathrm{~K}$ is usually used. To demonstrate the simplification method, the temperature is considered to ramp linearly with time $(t) . T=m t$, DExp becomes:

$$
D \operatorname{Exp}=\exp \left(-\int_{0}^{t} k_{0} e^{-\frac{E}{R m u}} d u\right)
$$

where:

$$
m \text { - heating rate }\left({ }^{\circ} \mathrm{C} \cdot \mathrm{min}^{-1}\right)
$$

The integral in the exponent can be approximated by using the Laplace approach, where the parameter $\frac{E}{R m t}$ is assumed to be large, hence the major contribution from the integral is when the value of $u$ is near $t$ or the temperature is near its maximum. This provides the well-known asymptotic approximation to DExp function:

$$
D \operatorname{Exp}=\exp \left(-\int_{0}^{t} k_{0} e^{-\frac{E}{R m u}} d u\right) \sim \exp \left(\frac{-k_{0} R m t^{2}}{E}\right) \text { as } \frac{E}{R m t} \rightarrow \infty \text { (3) }
$$

Eq. (3) can also be expressed in the form:

$$
D \operatorname{Exp}=\exp \left(-\exp \left(\frac{E_{s}-E}{E_{w}}\right)\right)
$$

where the function varies from zero to one as $E$ increases, over a range of step size $E_{w}$ around the central value $E_{s}$.

$$
\text { Let } h(E)=\left(\frac{E_{s}-E}{E_{w}}\right)
$$

After comparing it with Eq. (3), we get:

$$
h(E)=\frac{-E}{R m t}+\ln \left(\frac{k_{0} R m t^{2}}{E}\right)
$$

The behaviour of $E_{s}$ is of interest, so $h(E)$ is expanded by using Taylor series:

$$
h(E) \sim h\left(E_{s}\right)+\left(E-E_{s}\right) h^{\prime}\left(E_{s}\right)+\ldots
$$

Now $h(E), E_{s}$ and $E_{w}$ are chosen in such a manner:

$$
h\left(E_{s}\right)=0 \text { and } h\left(E_{s}\right)=\frac{-1}{E_{w}}
$$

After solving, we have:

$$
E_{s}=R m t Y\left(k_{0} t\right) \text { and } E_{w}=\frac{R m t E_{s}}{R m t+E_{s}}
$$

where:

$Y(x)$ - represents Lambert W function

\section{Distribution function}

There are two different limits which are applied to the distribution function $F(E)$. One, which is relatively wide initial distribution as compared with the width of DExp, is known as a wide initial distribution. If the initial distribution function is relatively narrow as compared with the width of DExp, it is defined as a narrow initial distribution. The significance of the distribution type defines the shape of the total integrand, which changes with time and applied limit. When the initial distribution is relatively wide compared to $E_{w}$ the total integrand initially behaves similar to the distribution $F(E)$. As time proceeds, it is truncated from the left by the step-like DExp. Moreover, the location of the maximum of the total integrand varies significantly and hence the shape becomes quite skewed. On the contrary, the total integrand remains similar in shape to the initial distribution:

$$
v=\int_{0}^{\infty} \frac{E}{\beta^{2}} \exp (g(E)) d E
$$

where:

$g(E)=-\exp \left(\frac{E_{s}-E}{E_{w}}\right)-\left(\frac{E}{\beta}\right)^{2}$ and $E_{s}$ and $E_{w}$ are functions of time $(t)$

Energy is now rescaled by $y=\frac{E}{E_{0}}$. So the problem becomes:

$$
\begin{gathered}
v=\frac{\pi}{2}\left(\int_{0}^{\infty} y \exp \left(-\exp \left(\frac{y_{s}-y}{y_{w}}\right)-\frac{\pi}{2} y^{2}\right) d y\right) \\
g(y)=-\exp \left(\frac{y_{s}-y}{y_{w}}\right)-\frac{\pi}{2} y^{2}
\end{gathered}
$$

For linear ramp temperature $T=m t$ :

$$
y_{s}=\frac{R m t Y\left(k_{0} t\right)}{E_{0}}, y_{w}=\frac{y_{s}}{1+Y\left(k_{0} t\right)}
$$

\section{Wide distribution case}

In this case, the initial distribution is much wider than DExp. To tackle this, the limit $\delta y_{w}<<1$ is considered. In this limit, DExp jumps from zero to one near $y=y_{s}$, as given previously (Howard, 1981; Vand, 1943; Pitt, 1962; Suuberg, 1983) and been approximated by the step-function.

$$
H\left(y-y_{s}\right)=\left\{\begin{array}{c}
1, y \geq y_{s} \\
0, y<y_{s}
\end{array}\right.
$$


Eq. (1) is rewritten in the form:

$$
\begin{aligned}
v= & \frac{\pi}{2} \int_{0}^{\infty} y\left[\exp \left(\left(-\exp \left(\frac{y_{s}-y}{y_{w}}\right)\right)\right)-H\left(y-y_{s}\right)\right] \\
& \exp \left(-\frac{\pi}{2} y^{2}\right) d y+\frac{\pi}{2} \int_{y_{s}}^{\infty} y \exp \left(-\frac{\pi}{2} y^{2}\right) d y
\end{aligned}
$$

or

$$
\begin{aligned}
v=\frac{\pi}{2} \int_{0}^{\infty} y\left[\exp \left(\left(-\exp \left(\frac{y_{s}-y}{y_{w}}\right)\right)\right)-H\left(y-y_{s}\right)\right] \\
\exp \left(-\frac{\pi}{2} y^{2}\right) d y+\left(1-C\left(y_{s}\right)\right)
\end{aligned}
$$

where:

$$
C\left(y_{s}\right)=1-\exp \left(-\frac{\pi}{2} y_{s}^{2}\right)
$$

Note that in the first integral, the integrand is the initial distribution multiplied by function which is very small everywhere but in a neighbourhood of size $y_{w}$ around the point $y=y_{s}$. Therefore, this integrand can be approximated by expanding the initial distribution.

Expanding the term $D(y)=y\left(\exp \left(-\frac{\pi}{2} y^{2}\right)\right)$ with the help of Taylor series about $y=y_{s}$ :

$$
\begin{gathered}
D(y) \sim D\left(y_{s}\right)+\left(y-y_{s}\right) D^{\prime}\left(y_{s}\right)+\frac{\left(y-y_{s}\right)^{2}}{2 !} D^{\prime \prime}\left(y_{s}\right)+ \\
+\frac{\left(y-y_{s}\right)^{3}}{3 !} D^{\prime \prime \prime}\left(y_{s}\right)
\end{gathered}
$$

or

$$
\begin{gathered}
D(y) \sim y_{s}\left(\exp \left(-\frac{\pi}{2} y_{s}^{2}\right)\right)\left(1-\frac{\left(y-y_{s}\right)}{y_{s}}\left(\pi y_{s}^{2}-1\right)+\pi \frac{\left(y-y_{s}\right)^{2}}{2 !}\right. \\
\left.\left.\left(\pi y_{s}^{2}-3\right)-\frac{\left(y-y_{s}\right)^{3}}{3 !} \pi\left(\left(\pi y_{s}^{2}\right)\right)^{2}-6 \pi y_{s}^{2}+3\right)\right)
\end{gathered}
$$

Substituting $x=\frac{y-y_{s}}{y_{w}}$, we get:

$$
\begin{gathered}
v=\frac{\pi}{2} \int_{0}^{\infty} y_{w}[\exp ((-\exp (-x)))-H(x)] y_{s}\left(\exp \left(-\frac{\pi}{2} y_{s}^{2}\right)\right) \\
\left(1-\frac{y_{w}}{y_{s}} x\left(\pi y_{s}^{2}-1\right)+\pi \frac{y_{w}^{2}}{2 !} x^{2}\left(\pi y_{s}^{2}-3\right)-\frac{y_{w}^{3} x^{3}}{3 ! y_{s}}\right. \\
\left.\left.\pi\left(\pi y_{s}^{2}\right)^{2}-6 \pi y_{s}^{2}+3\right)+\ldots\right) d x+\left(1-C\left(y_{s}\right)\right)
\end{gathered}
$$

Each of the integrals arising from a term in the Taylor series can be integrated separately to get the result for the first order reaction $(n=1)$ as:

$v \sim \frac{\pi}{2}\left(\exp \left(-\frac{\pi}{2} y_{s}^{2}\right)\right)\left(y_{w} y_{s} M_{0}-y_{w}^{2} M_{1}\left(\pi y_{s}^{2}-1\right)+\pi \frac{y_{w}^{3} y_{s}}{2} M_{2}\right.$ $\left(\pi y_{s}^{2}-3\right)-\frac{y_{w}^{4}}{6} \pi M_{3}\left(\left(\pi y_{s}^{2}\right)^{2}-6\left(\pi y_{s}^{2}+3\right)+\ldots\right)+\left(1-C\left(y_{s}\right)\right)$
The values of coefficient $M_{n}(n=0,1,2,3 \ldots)$ need only to be evaluated once, since they are independent of any parameters. The first few values are:

$$
M_{0} \approx-0.5772 M_{1} \approx-0.98906 M_{2} \approx-1.81496 M_{3} \approx-5.8903
$$

The values of the remaining coefficients are evaluated by integral:

$$
M_{n} \equiv \int_{-\infty}^{\infty} x_{i}\left(e^{-e^{-x}}-H(x)\right)
$$

Similarly, Eq. (7) for the $n^{\text {th }}$ order reaction is invoked. After simplification, we have:

$$
\begin{gathered}
v_{n^{t h}}=\int_{0}^{\infty}\left[1-\left(\exp \left(\frac{y_{s}-y}{y_{w}}\right)-H\left(y_{s}-y\right)\right)+\right. \\
\frac{n}{2}\left(\exp \left(2\left(\frac{y_{s}-y}{y_{w}}\right)\right)-H\left(y_{s}-y\right)\right)-\frac{(2 n-1)}{6} \\
\left.\left(\exp \left(3\left(\frac{y_{s}-y}{y_{w}}\right)\right)-H\left(y_{s}-y\right)\right)+\ldots\right] \\
\frac{\pi}{2} y_{s}\left(\exp \left(-\frac{\pi}{2} y_{s}^{2}\right)\right)\left(1-\frac{\left(y-y_{s}\right)}{y_{s}}\left(\pi y_{s}^{2}-1\right)+\right. \\
\left.\pi \frac{\left(y-y_{s}\right)^{2}}{2 !}\left(\pi y_{s}^{2}-3\right)-\frac{\left(y-y_{s}\right)^{3}}{y_{s} 3 !} \pi\left(\left(\pi y_{s}^{2}\right)^{2}-6 \pi y_{s}^{2}+3\right)\right) \\
d y+\left(\frac{n-5}{6}\right)\left(1-C\left(y_{s}\right)\right)
\end{gathered}
$$

Each of the integrals arising from a term in the Taylor series can be integrated separately to get the result for the $n^{\text {th }}$ order reaction $(n \neq 1)$ :

$$
\begin{gathered}
v_{n^{\text {th }}} \sim \frac{\pi}{2} \exp \left(-\frac{\pi}{2} y_{s}^{2}\right)\left[y_{w} y_{s}\left(P_{0}+\frac{n}{2} Q_{0}-\frac{(2 n-1)}{6} R_{0}\right)-\right. \\
y_{w}^{2}\left(P_{1}+\frac{n}{2} Q_{1}-\frac{(2 n-1)}{6} R_{1}\right)\left(\pi y_{s}^{2}-1\right)+\pi \frac{y_{w}^{3} y_{s}}{2} \\
\left(P_{2}+\frac{n}{2} Q_{2}-\frac{(2 n-1)}{6} R_{2}\right)\left(\pi y_{s}^{2}-3\right)-\frac{y_{w}^{4}}{6} \\
\left.\pi\left(P_{3}+\frac{n}{2} Q_{3}-\frac{(2 n-1)}{6} R_{3}\right)\left(\left(\pi y_{s}^{2}\right)^{2}-6 \pi y_{s}^{2}+3\right)\right]+ \\
\left(\frac{n-5}{6}\right)\left(1-C\left(y_{s}\right)\right)
\end{gathered}
$$

The first few values of $P_{n^{\prime}} Q_{n^{\prime}} R_{n}$ coefficients are:

$$
\begin{aligned}
& P_{0} \approx-0.36788 P_{1} \approx-0.23576 P_{2} \approx-0.17273 P_{3} \approx-0.13607 \\
& Q_{0} \approx-0.56767 Q_{1} \approx-0.35150 Q_{2} \approx-0.25250 Q_{3} \approx-0.19642 \\
& R_{0} \approx-0.68326 R_{1} \approx-0.41102 R_{2} \approx-0.29061 R_{3} \approx-0.22387
\end{aligned}
$$

The values of remaining coefficients are evaluated by the integrals as:

$$
P_{n} \equiv \int_{-\infty}^{\infty} x^{i}(\exp (-x)-U(x)) d x, i=0,1,2,3 \ldots
$$




$$
\begin{gathered}
Q_{n} \equiv \int_{-\infty}^{\infty} x^{i}(\exp (-2 x)-U(x)) d x, i=0,1,2,3 \ldots \\
R_{n} \equiv \int_{-\infty}^{\infty} x^{i}(\exp (-3 x)-U(x)) d x, i=0,1,2,3 \ldots
\end{gathered}
$$

\section{Narrow distribution case}

The approach in this study is focused on the same case of narrow distribution, but is incongruent from the approach of Bender and Orszag (1978) by incorporating the simultaneous change in the behaviour of the initial distribution $F(E)$. The term DExp is analogous to the method of the moving maximum for Laplace problems (Bender and Orszag, 1978). The method used by Bender and Orszag (1978) is to take the maximum of the total integrand by keeping the maximum of the initial distribution fixed. Here, the present approach provides results which are applicable to the wide range of operating parameters, since the maximum of integrand varies along with time.

Assume that $\sigma<R T$ for the linearly ramp temperature. Formally, the limit corresponding to the narrow distribution is $1<<\sigma y_{w}$. The value of $y_{e}$ of $y$ is evaluated, where the function $g(y)$ given in Eq. (5) is at an extremum by finding the point where $g\left(y_{e}\right)=0$. Hence:

$$
g\left(y_{e}\right)^{\prime}=\frac{1}{y_{w}} \exp \left(\frac{y_{s}-y}{y_{w}}\right)-\pi y_{e}
$$

It is necessary to find $y_{e}$ that would satisfy the Eq. (5):

$$
\exp \left(\frac{y_{s}-y_{e}}{y_{w}}\right)=\pi y_{w} y_{e}
$$

Finally, the solution is given by:

$$
y_{e}=y_{w} Y\left(\frac{1}{\pi y_{w}^{2}} \exp \left(\frac{y_{s}}{y_{w}}\right)\right)
$$

A Taylor series expansion $g(y)$ about $y_{e^{\prime}}$ truncated after quadratic terms, gives:

$$
g(y) \sim g\left(y_{e}\right)+\frac{\left(y-y_{e}\right)^{2} g^{\prime \prime}\left(y_{e}\right)}{2}+\ldots
$$

The second derivative of $g^{\prime \prime}(y)$ is obtained as:

$$
g(y)^{\prime \prime}=-\frac{1}{y_{w}^{2}} \exp \left(\frac{y_{s}-y_{e}}{y_{w}}\right)-\pi
$$

The leading behaviour of $v$ in Eq. (5) is:

$$
v \sim \frac{\exp \left(g\left(y_{e}\right)\right)}{\sqrt{\left|g^{\prime}\left(y_{e}\right)\right|}}
$$

This may also be written as:

$$
v \sim \frac{\exp \left[-\frac{\pi}{2} y_{e}\left(2 y_{w}+y_{e}\right)\right]}{\sqrt{\pi\left(1+\frac{y_{e}}{y_{w}}\right)}}
$$

Note: Applicability of Laplace method has failed in case of the $n^{\text {th }}$ order DAEM, as function $h^{\prime}\left(y_{e}\right)$ does not possess the real values of $y_{e} \forall \beta \in N$.

\section{Application of biomass}

The thermogravimetric data is obtained with the help of thermogravimetric analysis and differential thermal analysis in the presence of inert atmosphere of nitrogen. The experimental results have been used in the process of obtaining the $n^{\text {th }}$ order Rayleigh DAEM prediction through the asymptotic approximations. Table 1 shows the

\begin{tabular}{|c|c|c|c|c|c|c|c|}
\hline $\begin{array}{c}\text { C } \\
(\%)\end{array}$ & $\begin{array}{c}\text { H } \\
(\%)\end{array}$ & $\begin{array}{l}N \\
(\%)\end{array}$ & $\begin{array}{c}0 \\
(\%)\end{array}$ & S (\%) & $\begin{array}{l}\text { Ash } \\
\text { (\%) }\end{array}$ & $\begin{array}{l}\text { H.H.V* } \\
\left(\mathbf{k J} \cdot \mathbf{k g}^{-1}\right)\end{array}$ & $\begin{array}{l}\text { V.M** } \\
\text { (\%) }\end{array}$ \\
\hline 53.7 & 5.21 & 0.61 & 32.13 & 0.22 & 4.72 & 19.5 & 70.1 \\
\hline
\end{tabular}
chemical composition of pine needles, which is obtained through the ultimate analysis of samples of pine needles. The derived results of the paper have been implemented in the process of obtaining the $n^{\text {th }}$ order Rayleigh DAEM prediction. It is demonstrated in Fig. 6 that the $n^{\text {th }}$ order Rayleigh DAEM provided good fit with the experimental data.

Table 1 Chemical composition of pine needles

\section{Results and discusion}

In this study, the relevant parameters of biomass pyrolysis are determined. The effect of the frequency factor $\left(k_{0}\right)$ parameter on the numerical result is presented in Fig. 1. According to these curves, the increases in the value of $k_{0}$ lead the curves towards left. The observed decrease in the slope after inflexion indicates the increase in conversion rate increases as the increase in the frequency factor has been encountered. The influence of the upper limit $\left(E_{\infty}\right)$ of $\mathrm{d} E$ integral on the numerical results is shown in Fig. 2 . The observation reveals that in the initial phase of pyrolysis reaction, the remaining mass proportion $v$ must be close to one, whereas in Fig. 2, it is observed that the remaining mass fraction is less than 1 for $E<39.4 \mathrm{~kJ} \cdot \mathrm{mol}^{-1}$. With an increase in the value of $E$, the inflexion point approaches to zero, the mass fraction curves show asymptotic behaviour, and the releasing of volatile contents become saturated or maximized. When the values over $84.04 \mathrm{~kJ} \cdot \mathrm{mol}^{-1}$ are used for $E_{\infty}$ the results deviate drastically. The results are found to be corresponding to the thermoanalytical data for the range of $39.4 \leq E_{\infty}\left(\mathrm{kJ} \cdot \mathrm{mol}^{-1}\right)<84.04$. Therefore, the value $61.72 \mathrm{~kJ} \cdot \mathrm{mol}^{-1}$ can be used for the upper limit of the $\mathrm{d} E$ integral. The effect of heating rate on numerical results is illustrated in Fig. 3, where it can be seen that remaining mass fraction curves are shifted down the temperature scale with an increase in the heating rate $(m)$. The influence of the scale parameter of Rayleigh distribution on the numerical result is depicted in Fig. 4 . The increment in the value of scale parameter $(\beta)$ causes the increases in the slope of mass fraction curves. Moreover, the temperature scale is levitated with an increase in the value of $\beta$. At the value $\beta=10$, the results are found to be more accurate and closely proximate to each other. The effect of the reaction 
order $(n)$ values on the numerical results is shown in Fig. 5 , where it is observed that increase in $n$ values causes $v$ curves to lead toward the right direction.

The effect of limit imposed on the width of DExp, $y_{w}$ and the significance of time dependant maximum on the numerical results is depicted with the help of narrow distribution in Fig. 7 and Fig. 8. Relatively, narrow distribution has shown convergence for the increased values of frequency factor. The remaining mass fraction curves are shifted towards the left with an increase in the value of $k_{0}$. The influence of the upper limit of $d E$ is shown in Fig. 7 (b). For the value of $E_{\infty}>130 \mathrm{~kJ} \cdot \mathrm{mol}^{-1}$, the $v$ curves are close to one. With the increase in the value of $E_{\infty}$, the whole integrand proceeds similar to DExp. The results have shown accurate approximation for $E_{\infty} \leq 123.8 \mathrm{~kJ} \cdot \mathrm{mol}^{-1}$. Variation in numerical results with the change of heating rate is illustrated in Fig. 8(a). The remaining mass fraction curves lead towards the left with an increase in heating
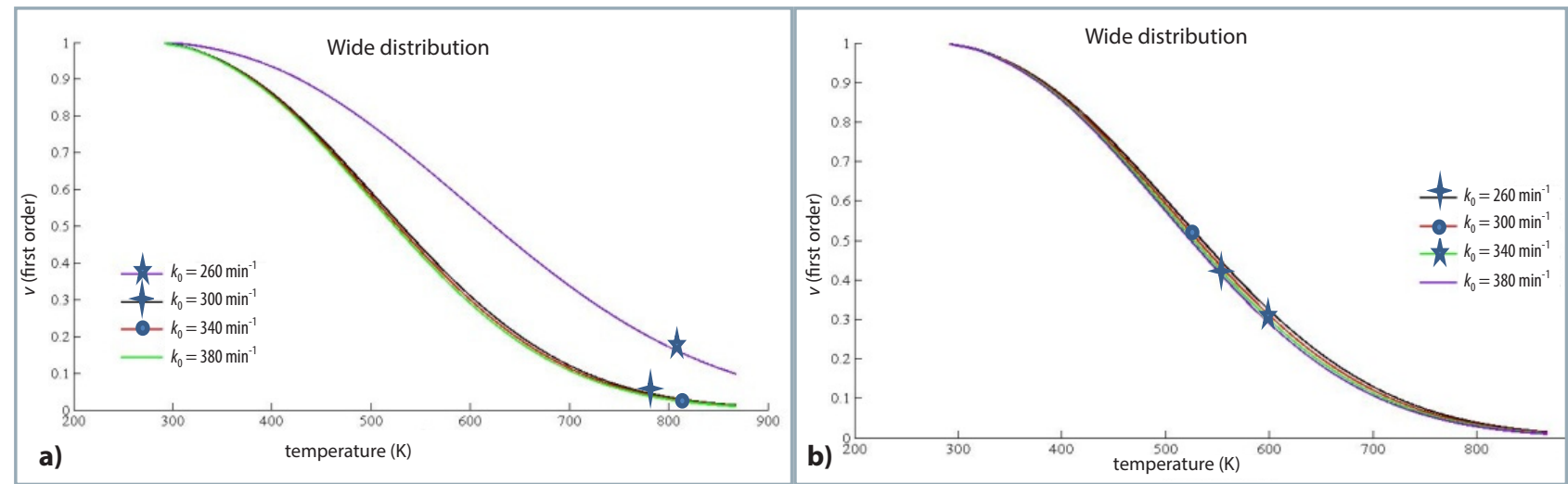

Fig. 1 The effect of frequency factor $\left(k_{0}\right)$ on the numerical results $\left(m=10^{\circ} \mathrm{C} \cdot \mathrm{min}^{-1}, T_{0}=293 \mathrm{~K}\right.$ and $\left.\beta=13\right)$ a) the first order, b) the $n^{\text {th }}$ order, $n=10.5$
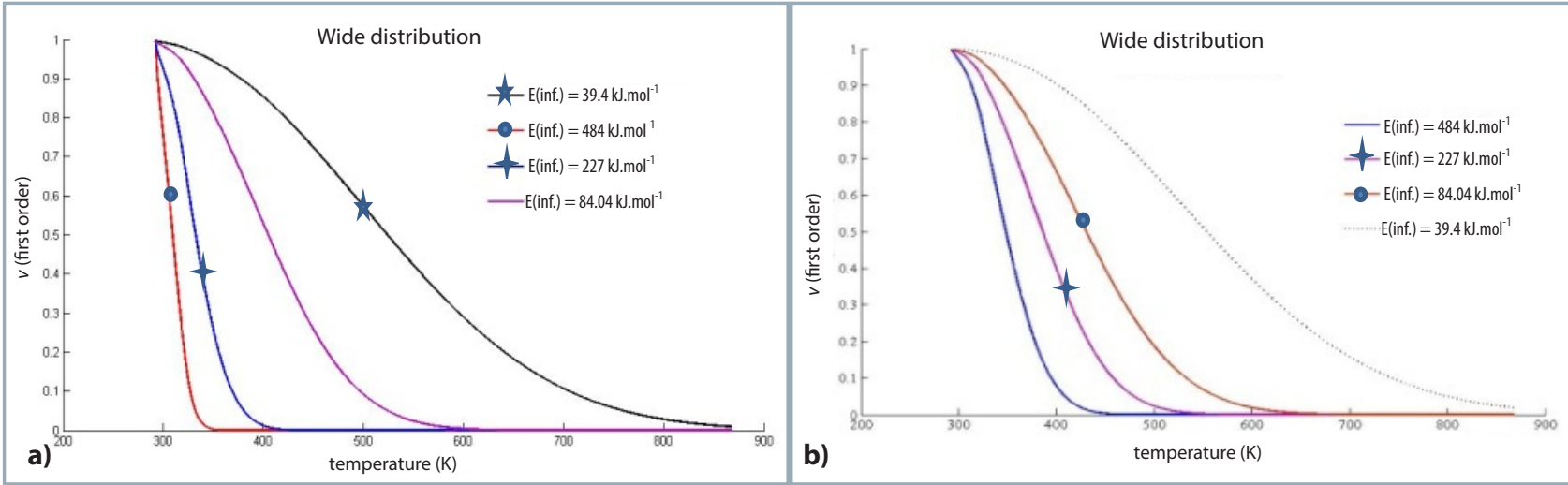

Fig. 2 The effect of the upper limit of $\mathrm{d} E$ integral on the numerical results $\left(k_{0}=250 \mathrm{~min}^{-1}, \mathrm{~m}=10^{\circ} \mathrm{C} \cdot \mathrm{min}^{-1}, T_{0}=293 \mathrm{~K}\right.$ and $\left.\beta=13\right)$ a) the first order; b) the $n^{\text {th }}$ order, $n=10.5$
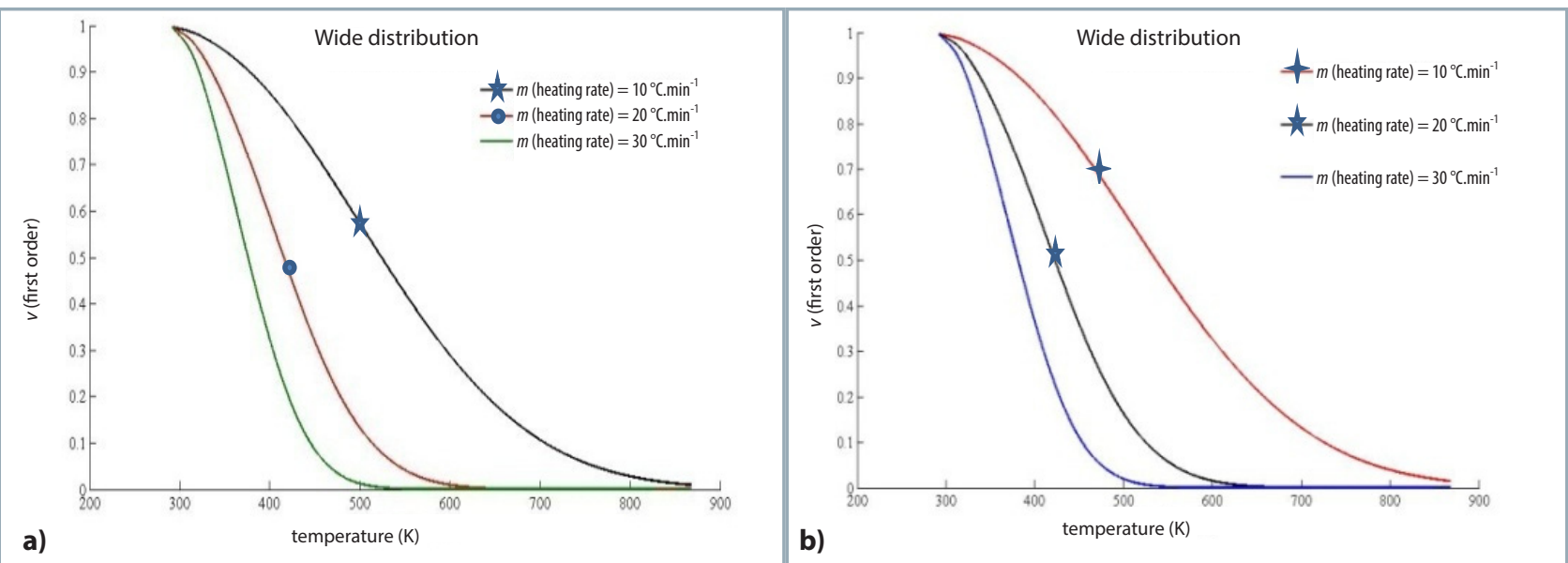

Fig. 3 The effect of heating rate $(m)$ on the numerical results $\left(k_{0}=250 \mathrm{~min}^{-1}, m=10^{\circ} \mathrm{C} \cdot \mathrm{min}^{-1}, T_{0}=293 \mathrm{~K}\right.$ and $\left.\beta=13\right)$ a) the first order, b) the $n^{\text {th }}$ order, $n=10.5$ 
rate. However, the effect of heating rate on the results obtained with the help of narrow distribution is similar to that of wide distribution for the first order and $n^{\text {th }}$ order. An increase in the value of the scale parameter causes the $v$ curves to shift up the temperature scale. Furthermore, the accurate results have been obtained for the lower values of the scale parameter of Rayleigh distribution. On the other hand, the results obtained by the symmetrical distribution functions (Cai et al., 2006; Dhaundiyal and Singh, 2016 a; Dhaundiyal and Singh, 2017; Dhaundiyal and Tewari, 2017) for the upper limit of activation energies $E_{\infty}\left(\mathrm{kJ} \cdot \mathrm{mol}^{-1}\right)$ are very close to the range of activation energies derived for the asymmetrical function, Rayleigh distribution. In case of another asymmetric function, Weibull distribution, the obtained value of activation energies differ from Rayleigh distribution through appreciable margin (Cai and Liu, 2007; Dhaundiyal and Singh, 2016 b). So, it is not necessary that the outcome of two asymmetrical functions may exhibit the same behaviour with the skewness of thermoanalytical data.

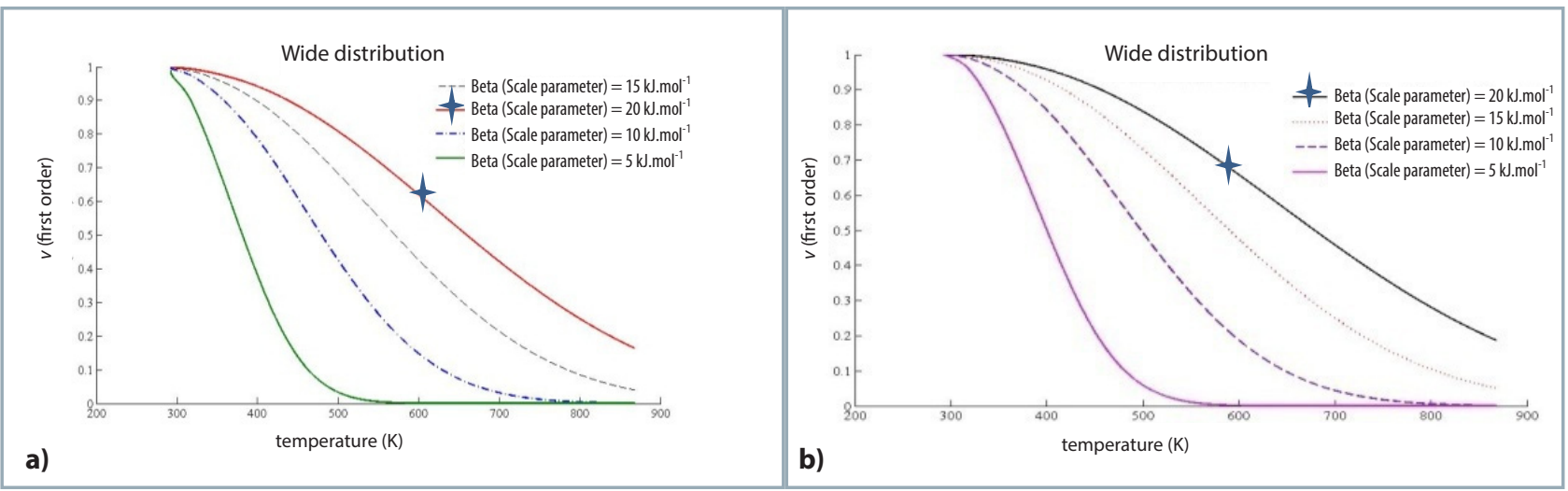

Fig. 4 The effect of the scale parameter of the Rayleigh distribution ( $\beta)$ on the numerical results $\left(k_{0}=250 \mathrm{~min}^{-1}, \mathrm{~m}=\right.$ $10^{\circ} \mathrm{C} \cdot \mathrm{min}^{-1}, T_{0}=293 \mathrm{~K}$ and $\beta=13$ )

a) the first order, b) the $n^{\text {th }}$ order, $n=10.5$

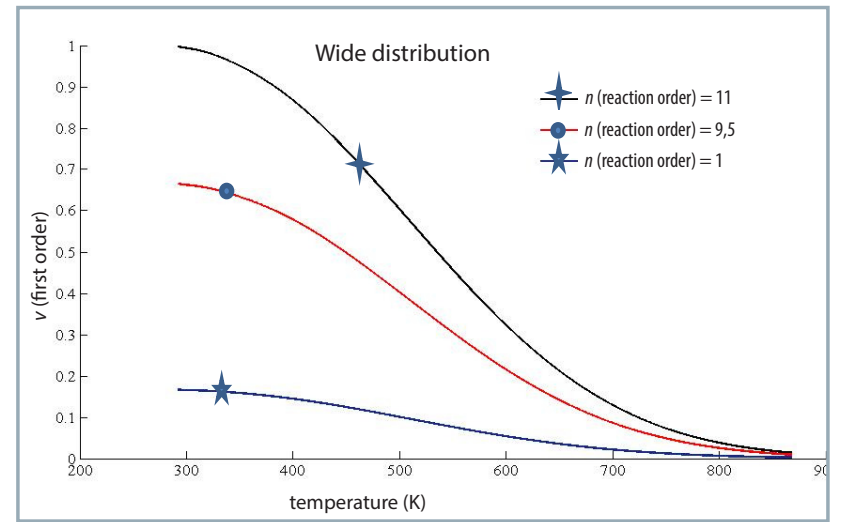

Fig. 5

The effect of reaction order $(n)$ on the numerical results $\left(k_{0}=250 \mathrm{~min}^{-1}, m=10^{\circ} \mathrm{C} \cdot \mathrm{min}^{-1}, T_{0}=293 \mathrm{~K}\right.$ and $\beta=13$ )

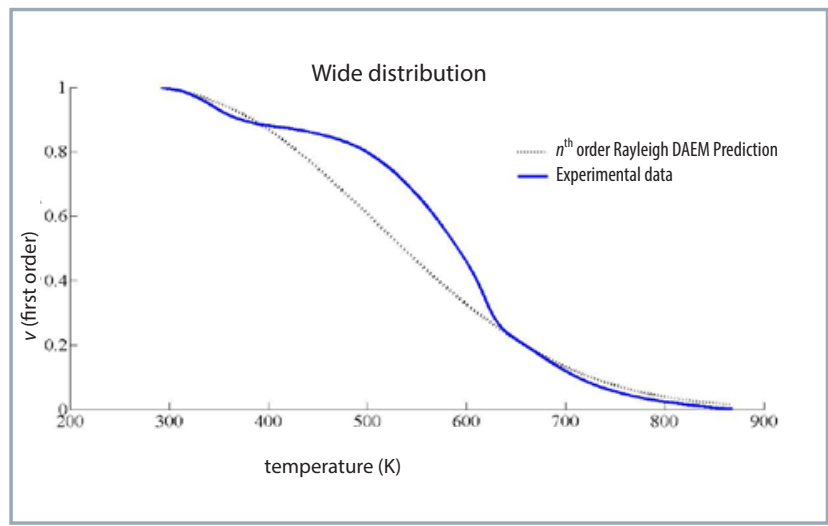

Fig. 6 Comparison between the experimental data and the $n^{\text {th }}$ order Rayleigh DAEM prediction

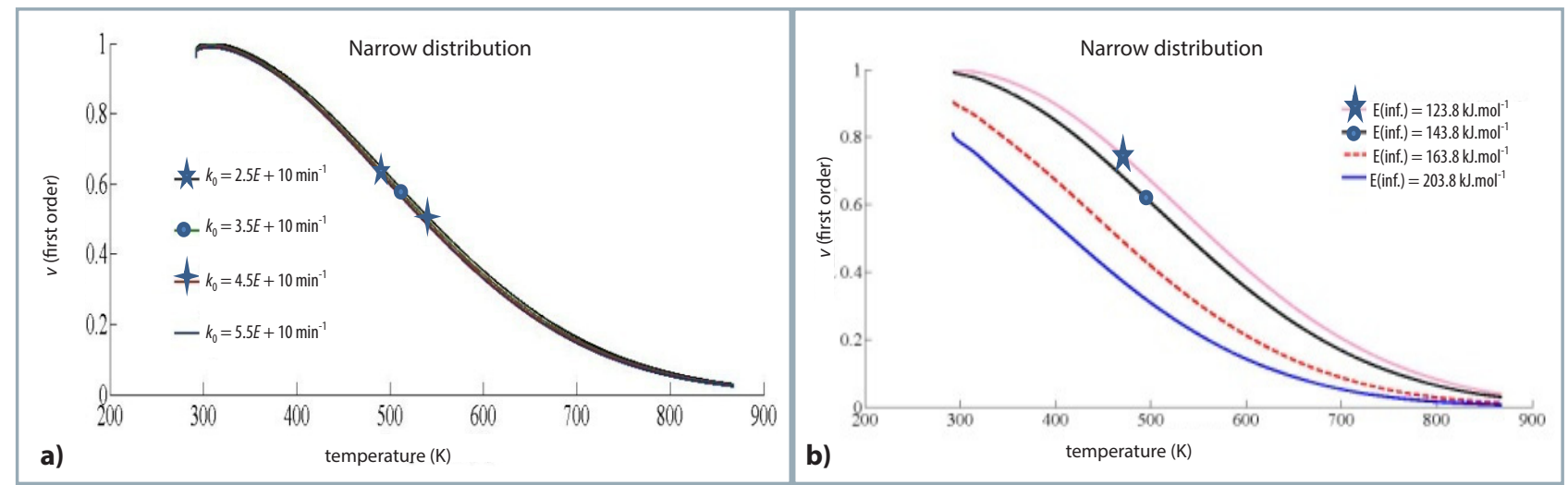

Fig. 7 a) The effect of frequency factor $\left(k_{0}\right)$ on the numerical results (narrow distribution)

b) The effect of upper limit of $\mathrm{d} E$ integral on the numerical results $\left(m=10^{\circ} \mathrm{C} \cdot \mathrm{min}^{-1}, T_{0}=293 \mathrm{~K}\right.$ and $\left.\beta=69\right)$ 


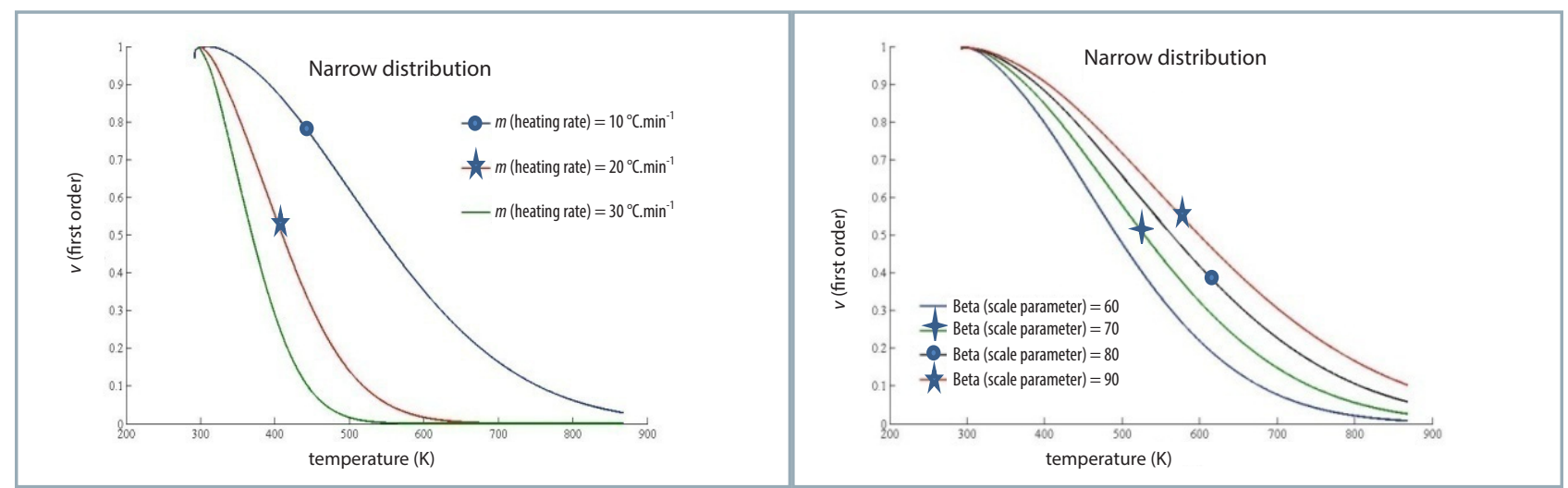

Fig. 8

a) The effect of heating rate $(m)$ on the numerical results $\left(k_{0}=2.4 e+10 \mathrm{~min}^{-1}, T_{0}=293 \mathrm{~K}\right.$ and $\left.\beta=69\right)$

b) The effect of the scale parameter of Rayleigh distribution on the numerical results $\left(k_{0}=2.4 e+10 \mathrm{~min}^{-1}, T_{0}=293 \mathrm{~K}\right.$ and $m=10^{\circ} \mathrm{C} \cdot \mathrm{min}^{-1}$ ) (narrow distribution)

\section{Conclusion}

The results have been obtained for different limits imposed on the numerical results with the help of asymptotic expansion. In order to find the parametric values for the numerical solutions of the non-isothermal $n^{\text {th }}$ order DAEM by means of Rayleigh distribution, the value $61.72 \mathrm{~kJ} \cdot \mathrm{mol}^{-1}$ can be used as the upper limit of the outer $\mathrm{d} E$ integral. The change in the values of heating rate, frequency factor, reaction order, and the scale parameter of Rayleigh distribution affects only the form of remaining mass fraction curves. The obtained results are a comprehensive overview of finding the boundary values for the relevant parameters of biomass pyrolysis. Some parameters are drastically varied with the change of the distribution type. The effect of DExp has also been studied, which provided us significant information about the upper cut-off limit for activation energies. For $E_{\infty}>123.8 \mathrm{~kJ} \cdot \mathrm{mol}^{-1}$, the solution has shown the exponential behaviour. The results are validated with other distribution function, and it has been found that the asymmetrical function, Rayleigh function, provided the most promising outcome for the non-isothermal condition, which is very close to the behaviour of symmetrical function. Although, it can be concluded that the range of activation energies obtained for the Rayleigh function is wider than that of Gaussian distribution for the isothermal condition (Dhaundiyal and Singh, 2017). On the comparative basis, the solution of the $n^{\text {th }}$ order reaction DAEM converged for the higher reaction orders than that of other distribution types (Dhaundiyal and Singh, 2016 a; Dhaundiyal and Singh, 2016 b; Dhaundiyal and Singh, 2017).

\section{References}

BENDER, C. M. - ORSZAG, S. A. 1978. Advanced Mathematical Methods for Scientists and Engineers. New York : Mc Graw Hill.

CAI, J. M. - FANG, H. - FUSHENG, Y. 2006. Nonisothermal $n^{\text {th }}$-order DAEM equation and its parametric study - Use in the kinetic analysis of biomass pyrolysis. In Journal of Mathematical Chemistry, vol. 42, no. 4, pp. 949-956.

CAI, J. M. - LIU, R. H. 2007. Parametric study of the nonisothermal $n^{\text {th }}$ order distributed activation energy model involved the Weibull distribution for biomass pyrolysis. In Journal of Thermal Analysis and Calorimetry, vol. 89, no. 3, pp. 971-975.
DHAUNDIYAL, A. - SINGH, S. B. 2016a. Distributed activation energy modelling for pyrolysis of forest waste using Gaussian distribution. In Proceedings of the Latvian Academy of Sciences. Section B. Natural, Exact, Applied Sciences, vol. 70, no. 1, pp. 64-70.

DHAUNDIYAL, A. - SINGH, S. B. 2016b. Asymptotic approximations to the distributed activation energy model for non isothermal pyrolysis of loose biomass using the Weibull distribution. In Archivum Combustionis, vol. 36, no. 2, pp. 131-146.

DHAUNDIYAL, A - SINGH, S. B. 2017. Parametric study of $n^{\text {th }}$ order distributed activation energy model for isothermal pyrolysis of forest waste using Gaussian distribution. In Acta Technologica Agriculturae, vol. 20, no. 1, pp. 23-28.

DHAUNDIYAL, A. - TEWARI, P. C. 2017. Kinetic parameters for the thermal decomposition of forest waste using distributed activation energy model (DAEM). In Environmental and Climate Technologies, vol. 19, pp. 15-32.

DONSKOI, E. - MCELWAIN, D. L. S. 1999. Approximate modelling of coal pyrolysis. In Fuel, vol. 78, no. 7, pp. 825-835.

HOWARD, J. B. 1981. Chapter 12. Fundamentals of coal pyrolysis and hydropyrolysis. In Chemistry of Coal Utilization. New York : John Wiley and Sons, pp. 665-784. ISBN 0471077267.

LAKSHMANAN, C. C. - WHITE, N. 1994. A new distributed activation energy model using Weibull distribution for the representation of complex kinetics. In Energy \& Fuels, vol. 8, no. 6, pp. 1158-1167.

NIKSA, S. - LAU, C.W. 1993. Global rates of devolatilization for various coal types. In Combustion abd Flame, vol. 94, no. 3, pp. 293-307.

PITT, G. J. 1962. The kinetics of the evolution of volatile products from coal. In Fuel, vol. 41, no. 1, pp. 267-274.

SUUBERG, E. M. 1983. Approximate solution technique for nonisothermal, Gaussian distributed activation energy models. In Combustion and Flame, vol. 50, pp. 243-245.

TENG, H. - HSIEH, C. T. 1999. Influence of surface characteristics on liquid-phase adsorption of phenol by activated carbons prepared from bituminous coal. In Industrial and Engineering Chemistry Research, vol. 37, no. 9, pp. 3618-3624.

VAND, A. 1943. Theory of the irreversible electrical resistance changes of metallic films evaporated in vacuum. In Proceedings of Physics Society of London A, vol. 55, pp. 222-246. 